\title{
Human Lateral Frontal Pole Contributes to Control over Emotional Approach-Avoidance Actions
}

\author{
(Dob Bramson, ${ }^{1}$ Davide Folloni, ${ }^{3,4}$ Lennart Verhagen, ${ }^{3,4}$ Bart Hartogsveld, ${ }^{5}$ Rogier B. Mars, ${ }^{1,4}$ Ivan Toni, ${ }^{1 *}$ \\ and ${ }^{\circ}$ Karin Roelofs ${ }^{1,2 *}$ \\ ${ }^{1}$ Donders Institute for Brain, Cognition and Behavior, Centre for Cognitive Neuroimaging, Radboud University Nijmegen, 6525 EN Nijmegen, The \\ Netherlands, ${ }^{2}$ Behavioral Science Institute, Radboud University Nijmegen, 6525 HR Nijmegen, The Netherlands, ${ }^{3}$ Wellcome Centre for Integrative \\ Neuroimaging, Department of Experimental Psychology, University of Oxford, Oxford OX1 3SR, United Kingdom, ${ }^{4}$ Wellcome Centre for Integrative \\ Neuroimaging, Centre for Functional MRI of the Brain, Nuffield Department of Clinical Neurosciences, John Radcliffe Hospital, University of Oxford, \\ Oxford OX3 9DU, United Kingdom, and 5Department of Clinical Psychological Science, Faculty of Psychology and Neuroscience, Maastricht University, \\ 6229 ER Maastricht, The Netherlands
}

Regulation of emotional behavior is essential for human social interactions. Recent work has exposed its cognitive complexity, as well as its unexpected reliance on portions of the anterior PFC ( $\mathrm{aPFC}$ ) also involved in exploration, relational reasoning, and counterfactual choice, rather than on dorsolateral and medial prefrontal areas involved in several forms of cognitive control. This study anatomically qualifies the contribution of aPFC territories to the regulation of prepotent approach-avoidance action tendencies elicited by emotional faces, and explores a possible structural pathway through which this emotional action regulation might be implemented. We provide converging evidence from task-based fMRI, diffusion-weighted imaging, and functional connectivity fingerprints for a novel neural element in emotional regulation. Task-based fMRI in human male participants $(N=40)$ performing an emotional approach-avoidance task identified aPFC territories involved in the regulation of action tendencies elicited by emotional faces. Connectivity fingerprints, based on diffusion-weighted imaging and resting-state connectivity, localized those task-defined frontal regions to the lateral frontal pole (FPl), an anatomically defined portion of the aPFC that lacks a homologous counterpart in macaque brains. Probabilistic tractography indicated that $10 \%-20 \%$ of interindividual variation in emotional regulation abilities is accounted for by the strength of structural connectivity between FPl and amygdala. Evidence from an independent replication sample $(N=50 ; 10$ females $)$ further substantiated this result. These findings provide novel neuroanatomical evidence for incorporating FPl in models of control over human action tendencies elicited by emotional faces.

Key words: amygdalofugal connectivity; approach-avoidance; lateral frontal pole; prefrontal control; social-emotional action control

\section{Significance Statement}

Successful regulation of emotional behaviors is a prerequisite for successful participation in human society, as is evidenced by the social isolation and loss of occupational opportunities often encountered by people suffering from emotion regulation disorders, such as social-anxiety disorder and psychopathy. Knowledge about the precise cortical regions and connections supporting this control is crucial for understanding both the nature of computations needed to successfully traverse the space of possible actions in social situations, and the potential interventions that might result in efficient treatment of social-emotional disorders. This study provides evidence for a precise cortical region (lateral frontal pole) and a structural pathway (the ventral amygdalofugal bundle) through which a cognitively complex form of emotional action regulation might be implemented in the human brain.

\section{Introduction}

Control over emotional behavior is paramount for successful participation in society (Hare, 2017). However, regulating emo-

Received Aug. 19, 2019; revised Jan. 17, 2020; accepted Jan. 17, 2020.

Author contributions: B.B., R.B.M., I.T., and K.R. designed research; B.B. and B.H. performed research; B.B., D.F.,

L.V., R.B.M., I.T., and K.R. analyzed data; B.B., I.T., and K.R. wrote the first draft of the paper; B.B., D.F., L.V., R.B.M.,

I.T., and K.R. edited the paper; B.B., I.T., and K.R. wrote the paper. tional actions is cognitively complex. It requires the ability to assess the effectiveness of ongoing behavior and compare this with alternative action strategies. For instance, a scientist present-
This work was supported by The Netherlands Organization for Scientific Research VICI Grant 453-12-001 and European Research Council Consolidator Grant ERC_CoG-2017_772337 to K.R. R.B.M. was supported by The Netherlands Organization for Scientific Research Grant 452-13-015. D.F. was supported by Wellcome Trust UK Grant 105238/Z/14/Z. 
ing work in front of a critical audience needs to overcome the tendency to avoid potential criticism to reap the long-term benefits of peer exposure and constructive feedback. Recent converging evidence indicates that this type of regulation of emotional behavior is implemented by the rostral part of the anterior PFC (aPFC) (Volman et al., 2011a, 2013; Koch et al., 2018). The current study anatomically qualifies the contribution of aPFC regions to the regulation of emotional action tendencies elicited by social-emotional cues, and explores a structural pathway through which this regulation might be implemented.

The aPFC has been involved in a disparate range of cognitive tasks, for example, relational reasoning (Vendetti and Bunge, 2014; Hartogsveld et al., 2018), counterfactual choice (Boorman et al., 2009; Mansouri et al., 2017), and exploration behavior (Daw et al., 2006; Zajkowski et al., 2017). To date, emotional action regulation has not been considered part of the cognitive fingerprint of this region (Koch et al., 2018). Models of emotion regulation have predominantly focused on dlPFC and mPFC (Etkin et al., 2015; Morawetz et al., 2017; Langner et al., 2018). These models are largely based on explicit regulation strategies (e.g., reappraisal) (Wager et al., 2008; Buhle et al., 2014) and have ignored control of emotional actions, an important component of emotion regulation concerned with conflict between the emotional value of stimulus and response (Frijda et al., 2014; Ridderinkhof, 2017; Bramson et al., 2018). At first glance, the focus of the emotion control literature on mPFC is justified by strong structural and functional connectivity between $\mathrm{mPFC}$ and areas involved in emotional and social processing, such as the amygdala (Ghashghaei and Barbas, 2002; Petrides and Pandya, 2007; Neubert et al., 2014; Tillman et al., 2018). In contrast, structural connections to the amygdala are scarce for anterior prefrontal areas (Ghashghaei et al., 2007). However, recent studies have shown that the amygdala is connected to the aPFC via rostral projections of the ventral amygdalofugal pathway (Krüger et al., 2015; Kamali et al., 2016). Indeed, there is evidence that this tract might extend to the lateral frontal pole (FPl) (Folloni et al., 2019), a portion of the human aPFC that does not have a homological counterpart in macaque brains (Neubert et al., 2014). These anatomical observations, together with converging functional evidence on aPFC involvement in the regulation of emotional strategies and actions (Bramson et al., 2018; Koch et al., 2018), raise the possibility that human emotional action control is coordinated by the FPl.

In this study, we combine evidence from task-based fMRI, diffusion-weighted imaging (DWI), and resting-state connectivity fingerprints to define which portion of aPFC supports emotional action control. First, we characterize the functional specificity of aPFC contributions to emotional action control through a social-emotional approach-avoidance (AA) task. Participants approached or avoided happy and angry faces by means of speeded joystick reactions. Approaching angry and avoiding happy faces requires control over habitual emotional action tendencies and elicits activity in aPFC (Roelofs et al., 2009a; Volman et al., 2011b). The social relevance of the emotional control indexed by this task has been established in social-emotional disorders (Volman et al., 2016; Bertsch et al., 2017) and healthy individuals where it predicted responses to real-life social stress

The authors declare no competing financial interests.

*I.T. and K.R. contributed equally to this work.

Correspondence should be addressed to Bob Bramson at b.bramson@donders.ru.nl.

https://doi.org/10.1523/JNEUROSCI.2048-19.2020

Copyright $(2020$ the authors induction (Kaldewaij et al., 2019a). Second, we anatomically localize aPFC territories activated by the AA task by matching structural and functional connectivity fingerprints of this area to fingerprints extracted from known areas of the aPFC (Neubert et al., 2014). Third, we assess the evidence for, and the functional relevance of, a structural pathway through which the portion of the aPFC activated by the AA task might support emotional action regulation, namely, through direct connections with the amygdala (Folloni et al., 2019).

\section{Materials and Methods}

Participants. Forty male students from Radboud University Nijmegen participated in this study after giving informed consent. The sample had a mean age of 23.5 years (SD 2.8 years, range $18-33$ years). None of the participants reported history of mental illness or use of psychoactive/ corticosteroid medication. All had normal or corrected-to-normal vision, and were screened for counterindications for MRI. The study was approved by the local ethics committee (CMO:2014/288). Sample size was based on behavioral congruency effects observed previously in the same task (effect size $d=0.4$ ) (Volman et al., 2011a). The sample consisted of males only to minimize potential variability caused by sex differences and fluctuations in cortisol and testosterone (e.g., due to different phases in the menstrual cycle). These hormones are known to influence behavioral and neural responses on the AA task (van Peer et al., 2007; Volman et al., 2011b; Kaldewaij et al., 2019a), and controlling for those fluctuations would have required a larger sample.

Given the novelty of one of the main findings of this report (correlation between amygdalofugal-FPl connectivity and behavioral congruency effect; see Results), we set out to replicate that finding in an independent convenience sample consisting of 50 participants (10 females; mean age 23.8 years, SD 3.4 years, range $18-34$ years). These participants took part in another study where they performed the AA task in a similar test context, as well as a DWI session. This second study was performed as part of a larger research project, after the analyses of the current study were finalized.

Materials and apparatus. Images were acquired using a $3 \mathrm{~T}$ MAGNETROM Prisma MRI scanner (Siemens AG) using a 32 channel head coil for functional and DWI images and a 20 channel head coil for structural T1 images. Stimuli were presented using an EIKI LC-XL100 beamer with a resolution of $1024 \times 768$ and a refresh rate of $60 \mathrm{~Hz}$, and were projected onto a screen behind the scanner bore. Participants were able to see the screen via a mirror.

The AA task consisted of 16 blocks of 12 trials each, in which participants had to approach or avoid equiluminant happy or angry faces presented in the center of the screen. Faces were presented for $100 \mathrm{~ms}$ and preceded by a $500 \mathrm{~ms}$ fixation cross in the center of the screen. After the presentation of the face, participants had $2000 \mathrm{~ms}$ to respond using a joystick. Each trial was followed by an intertrial interval of 2-4 s. Task instructions for congruent (move the joystick toward you/away from you for happy/angry faces) and incongruent blocks (move the joystick toward you/away from you for angry/happy faces) were presented before each block and switched between blocks. Subjects responded using a customized fiber optic response joystick, which was fixed to only move in the sagittal plane. Affect-incongruent trials have consistently been shown to activate the aPFC (Roelofs et al., 2009a; Volman et al., 2011a; Tyborowska et al., 2016; Kaldewaij et al., 2019a). Behavioral and neural responses on this task are affected in several social-emotional disorders, such as psychopathy, social-anxiety, and borderline disorder (Heuer et al., 2007; von Borries et al., 2012; Roelofs and Cremers, 2015; Bertsch et al., 2018), and those responses are influenced by hormones affecting social behavior (Volman et al., 2016; Kaldewaij et al., 2019a).

Procedure. Data were acquired on two different days; day 1 consisted of the AA task, resting-state, and T1 structural scan. DWI was acquired on day 2. Before and after the AA task, we took saliva measurements to possibly control for testosterone and cortisol for other research purposes. On day 2, each participant filled out the State Trait Anxiety Inventory to control for effects of trait anxiety. 
Functional scans. The FOV of the scans acquired in the two MR sessions was aligned to a built-in brain atlas using an auto-align head scout sequence. During resting-state and task fMRI sessions, we acquired BOLD-sensitive images using a multiband sequence with $\mathrm{TR}=735 \mathrm{~ms} /$ $\mathrm{TE}=39 \mathrm{~ms}, 64$ slices, flip angle of $52^{\circ}$, multiband acceleration factor of 8 , slice orientation $\mathrm{T}>\mathrm{C}$, voxel size $=2.4 \times 2.4 \times 2.4 \mathrm{~mm}$, phase encoding direction $\mathrm{A} \gg \mathrm{P}$. The resting-state session lasted $8.5 \mathrm{~min}$ (700 images). Each sequence was followed by a field map image; flip angle $=$ $60^{\circ}, \mathrm{TR}=614, \mathrm{TE}=4.92$.

Structural scan. High-resolution anatomical images were acquired with a single-shot MPRAGE sequence with an acceleration factor of 2 (GRAPPA method), a TR of $2400 \mathrm{~ms}$, TE $2.13 \mathrm{~ms}$. Effective voxel size was $1 \times 1 \times 1 \mathrm{~mm}$ with 176 sagittal slices, distance factor $50 \%$, flip angle $8^{\circ}$, orientation $\mathrm{A} \gg$ P, FOV $256 \mathrm{~mm}$.

DWI. Diffusion-weighted images were acquired using EPI with an acceleration factor of 2 (GRAPPA method). We acquired 652 -mm-thick axial slices, with a voxel size of $2 \times 2 \times 2 \mathrm{~mm}$, phase encoding direction $\mathrm{A} \gg$ P, FOV $220 \mathrm{~mm}$. In addition, we acquired 10 volumes without diffusion weighting $\left(\mathrm{b}=0 \mathrm{~s} / \mathrm{mm}^{2}\right), 30$ isotropically distributed directions using a b value of $750 \mathrm{~s} / \mathrm{mm}^{2}$, and 60 isotropically distributed directions a b value of $3000 \mathrm{~s} / \mathrm{mm}^{2}$. We also acquired a volume without diffusion weighting with reverse phase encoding $(\mathrm{P} \gg \mathrm{A})$.

Diffusion-weighted images for the replication sample were acquired using EPI with multiband acceleration factor of 2 (GRAPPA method), multiband acceleration factor $=3$. For this dataset, we acquired 931.6 $\mathrm{mm}$-thick transversal slices with voxel size of $1.6 \times 1.6 \times 1.6 \mathrm{~mm}$, phase encoding direction $\mathrm{A} \gg \mathrm{P}, \mathrm{FOV} 211 \mathrm{~mm}, \mathrm{TR}=3350, \mathrm{TE}=71.20 ; 256$ isotropically distributed directions were acquired using a b value of 2500 $\mathrm{s} / \mathrm{mm}^{2}$. We also acquired a volume without diffusion weighting with reverse phase encoding $(\mathrm{P} \gg \mathrm{A})$. Preprocessing and analyses for this dataset were the same as for the original sample (reported below).

Behavioral analyses. Behavioral analyses of AA task performance contrasted incongruent to congruent trials, comparing reaction times and percentage correct between conditions. Differences between conditions were assessed using repeated-measures ANOVA and paired-sample $t$ tests implemented in JASP (version 0.9.2; https://jasp-stats.org/).

Analyses: functional images. All processing of the AA and resting-state images was done using MELODIC 3.00 as implemented in FSL 5.0.10 (https://fsl.fmrib.ox.ac.uk). Images were motion-corrected using MCFLIRT (Jenkinson et al., 2002), and distortions in the magnetic field were corrected using fieldmap correction in FUGUE. Functional images were rigid-body registered to the brain-extracted structural image using FLIRT. Registration to MNI $2 \mathrm{~mm}$ standard space was done using the nonlinear registration tool FNIRT. Images were spatially smoothed using a Gaussian $5 \mathrm{~mm}$ kernel and low passed filtered with a cutoff at $100 \mathrm{~s}$. Independent component analysis was run (Beckmann and Smith, 2004), after which the first 10 components were manually inspected to remove sources of noise (Griffanti et al., 2017).

First- and second-level analyses were done in FEAT 6.00 implemented in FSL 5.0.10 (https://fsl.fmrib.ox.ac.uk/fsl/fslwiki/) (Woolrich et al., 2001, 2004). We set up separate GLMs for each task. The first-level AA model consisted of four task regressors: approach angry, approach happy, avoid angry, and avoid happy; six motion regressors and their temporal derivatives; and two regressors modeling fluctuations in signal in white matter and CSF. Approach happy and avoid angry (congruent conditions) were then contrasted with approach angry and avoid happy (incongruent conditions). This contrast was taken as input for each subject in the second-level analysis. Second-level analysis was performed using Centre for Functional MRI of the Brain's Local Analysis of Mixed Effects (FLAME 1) with outlier deweighting. We used a cluster forming threshold of $Z>2.3$, a value that has been shown to effectively control for family-wise error rate, possibly related to the fact that FLAME estimates and takes into account within-subject variance (Eklund et al., 2015). Locations with above-threshold activity were localized using masks for the PFC (Sallet et al., 2013; Neubert et al., 2014), parietal cortex (Mars et al., 2011), and whole-brain cortical and subcortical atlases (HarvardOxford Atlas; Desikan et al., 2006).

To assess psychophysiological interactions during the AA task, we ran a second analysis using FEAT with regressors representing the task effects of interest (incongruent-congruent), the regressor incongruent + congruent, a regressor describing the first eigenvariate time series of activation of the seed region: bilateral amygdalae taken from the AAL atlas (Tzourio-Mazoyer et al., 2002), and a regressor describing the interaction between the seed region activity and task effects. Strength of amygdalofugal-FPl connections as estimated from tractography of dwMRI (see below) was added as a covariate in the second-level analysis.

Analyses: functional connectivity fingerprints of FPl based on restingstate fMRI. For the resting-state fMRI analysis, we defined ROIs for each of the three areas in aPFC (FPl, FPm, and area 46), as identified by Neubert et al. (2014). These masks were thresholded to contain only the $25 \%$ of voxels that were most likely to be part of each area (Mars et al., 2016; Hartogsveld et al., 2018). The masks were warped to each individual anatomical space, after which we extracted the first eigenvariate time series from each area. These time series were correlated with whole-brain resting-state activity, the results of which were transformed to $z$ values using Fishers $z$-transform and normalized. Resulting correlation maps were transformed back to MNI space, after which we extracted the connectivity from each prefrontal region with five downstream regions: posterior cingulate cortex (PCC) $[10,-52,24]$, intraparietal lobule [48, $-46,48]$, temporal pole $[34,12,-36]$, ventromedial PFC (vmPFC) [8, $44,-14]$, and dIPFC $[46,20,40]$, for each hemisphere separately. Differential connectivity with these five areas has previously been shown to be appropriate to dissociate different cortical regions within aPFC (Mars et al., 2016; Hartogsveld et al., 2018). This procedure creates a "connectivity fingerprint" of each region, describing functional (and structural for DWI analyses) connections with other brain areas, and rests on the premise that each region's unique functional repertoire is determined by its connections with other regions (Passingham et al., 2002; Mars et al., 2018). We followed the same procedure for the two regions found in the aPFC during the AA task: $5 \mathrm{~mm}$ sphere at approximately MNI [24, 50, $-4]$ and MNI $[-24,52,4]$. The focus of this study on aPFC responses to congruency demands during performance of the AA task is related to a large body of work that has characterized and replicated those aPFC responses across numerous studies (Roelofs et al., 2009a; Volman et al., 2011a; Tyborowska et al., 2016). That work has shown that activity in aPFC during incongruent trials is correlated with, and necessary for, successfully overriding automatic action tendencies (Volman et al., 2011a); is influenced by several neuromodulators, such as testosterone and cortisol (van Peer et al., 2007; Volman et al., 2011b, 2016; Kaldewaij et al., 2019a); is augmented in social psychopathology (von Borries et al., 2012; Radke et al., 2013; Bertsch et al., 2017, 2018); and predicts realworld stress coping (Kaldewaij et al., 2019b). The added value and validity of the structural explanation provided in the current study depend on using the same functional comparison implemented in those previous studies, namely, a direct contrast between incongruent and congruent conditions.

For the statistical analysis, we computed the "city block" or "Manhattan" distance between the connectivity profiles of the AA regions and the connectivity profiles of the three prefrontal masks, separately for each hemisphere. This measure consists of the sum of absolute distance between different connectivity profiles and has previously been used to describe the relative dissimilarity between different connectivity profiles (Sallet et al., 2013; Neubert et al., 2014; Mars et al., 2016). We compared these distance measures to a distribution of randomized distances that we created by permuting the region labels 10000 times, each time randomly swapping the target region labels (FPl, FPm, area 46) within participants and computing the Manhattan distance with the AA fingerprint. The result of this approach shows whether the connectivity profile of the aPFC regions differs from the connectivity profile of the region active during the AA task (Mars et al., 2016).

Analyses: structural connectivity fingerprints of FPL based on diffusionweighted MRI. All analyses of diffusion data were performed in FSL FDT 3.0 (https://fsl.fmrib.ox.ac.uk). We used TOPUP to estimate susceptibility artifacts using additional $\mathrm{b}=0$ volumes with reverse phase coding direction (Andersson et al., 2003). Next, we used EDDY (using the fieldmap estimated by TOPUP) to correct for distortions caused by eddy currents and subject movement (Andersson and Sotiropoulos, 2016). 
We used BedpostX to fit a crossing fiber model using default settings (Behrens et al., 2007).

Seed regions were defined for five major tracts by creating $5 \mathrm{~mm}$ spheres in the cingulum bundle (CB), MNI: $[8,-2,36]$; superior longitudinal fasciculus 1 (SLF1), MNI: [16, $-2,54]$; SLF 2, MNI: [30, -2, 38]; SLF 3, MNI: $[44,-2,26]$; and uncinate fasciculus (UF), MNI: $[32,-2,-10]$ and then warped to individual subject space. As with the functional connectivity described above, these specific fiber bundles were chosen because they have been shown to be differentially connected to distinct areas within the aPFC (Neubert et al., 2014; Mars et al., 2016; Hartogsveld et al., 2018). The procedure for amygdalofugal connectivity is described below. For the comparisons in the left hemisphere, the same coordinates were used with the $x$ axis coordinate inverted. ProbtrackX was run with default settings using these masks as seed, separately for each hemisphere using an exclusion mask at $x=0$. The results from probtrackX were warped to MNI $1 \mathrm{~mm}$ space, log-transformed, and normalized to the highest probability per tract to allow a direct comparison between tracts with all values ranging between 0 and 1 . We computed connectivity profiles of $\mathrm{FPl}$, FPm, and area 46 by counting the number of times each tract ended in these regions. These connectivity profiles were compared with the connectivity profile of the region in the aPFC resulting from the AA task contrast $(5 \mathrm{~mm}$ sphere at MNI: $[24,50,-4]$ for right and MNI: $[-24,52,4]$ for left). We computed Manhattan distance between the connectivity profiles of the AA task and the three prefrontal masks and permuted this 10,000 times, each time randomly swapping the target region labels (FPl, FPm, and area 46) within participants.

Analyses: amygdalofugal connectivity. Connections between amygdala and aPFC were reconstructed using probtrackX. For this, we used the procedure and masks provided by Folloni et al. (2019). We seeded these connections in the white matter punctuating the extended amygdala and substantia innominata: MNI: $[-7,3,-9]$ and an all-coronal waypoint mask at $y=22$. We used tractography masks to ensure the estimated seedlines extended only rostrally, at least up to the $y=25$ coronal plane, and excluded CSF and between hemisphere connections. Connection strength was normalized and log-transformed within each participant. Next, we extracted the total amount of times the tractography entered the FPl, FPm, and area 46 ROIs separately. These values were correlated with congruency effects on reaction time and percentage correct using Spearman's correlation coefficient.

To exclude that the amygdalofugal-FPl connections are influenced by (much stronger) projections of the UF), we reconstructed UF connectivity with $\mathrm{FPl}$ and correlated this with the behavioral congruency. To isolate the UF, we placed a seed in the white matter rostrolaterally to the amygdala (MNI: $[35,2,-22]$ ), a coronal waypoint mask at $y=22$ and exclusion masks in the CSF and between hemispheres. We also used partial correlation to regress out possible correlations influences of UF and state anxiety (State Anxiety Inventory-Y2) in the amygdalofugalbehavioral correlations. Finally, we used an independent comparison sample to confirm the correlation between amygdalofugal-FPl connectivity and behavioral congruency.

\section{Results}

The study had two main goals: (1) localize aPFC responses evoked during control over action tendencies elicited by emotional faces in relation to an established anatomical parcellation
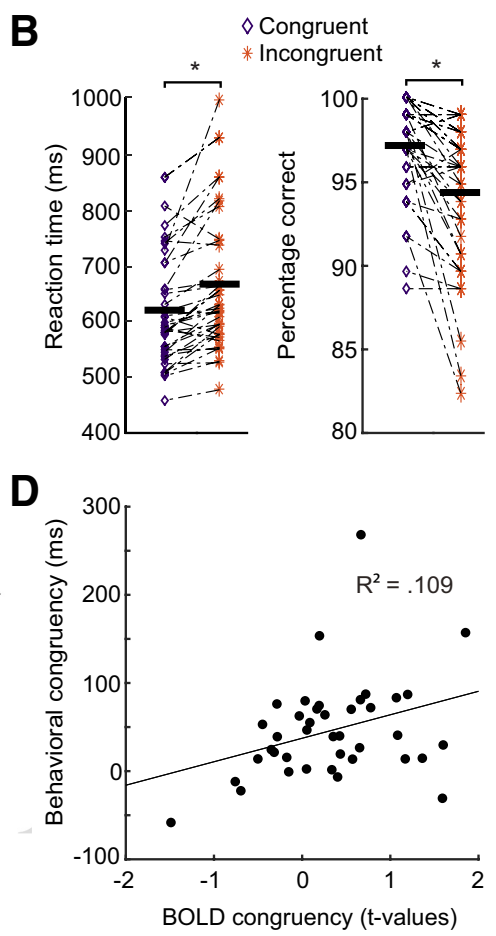
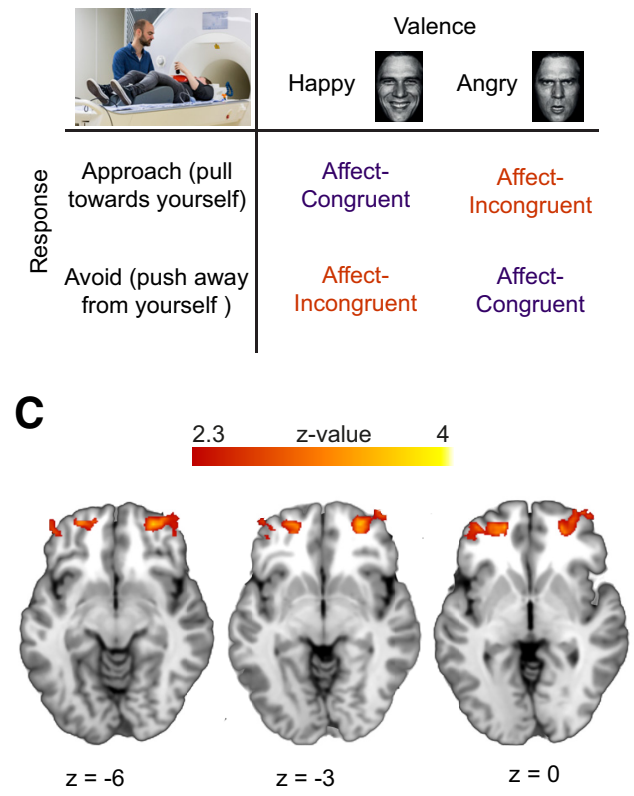

Figure 1. Effects of cognitive control over social-emotional action tendencies. $\boldsymbol{A}$, Schematic representation of the AA task used ave control over social-emotional action tendencies. The incongruent condition requires control to override the automatic

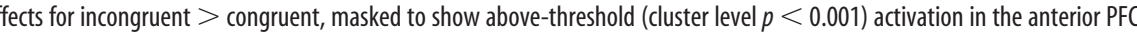
frontal pole mask; Harvard-0xford Atlas; Desikan et al., 2006). D, Correlation between reaction time congruency and BOLD congruency effects extracted from the FPI across participants.

of the aPFC; and (2) provide evidence for the functional relevance of amygdala-aPFC connectivity in implementing control over emotional actions. We implemented $a$ priori determined analyses addressing these two goals, correcting the statistical inferences for multiple comparisons, either through cluster correction (fMRI task contrasts) or Bonferroni-correction (anatomical localization and tract-behavioral correlations). Furthermore, we implemented a number of follow-up exploratory analyses, prioritizing sensitivity over specificity, thus avoiding corrections for multiple comparisons. The exploratory nature of these analyses is marked in the text.

\section{AA task: behavioral costs of controlling emotional behavior} There were interaction effects between emotion and movement for reaction times $\left(F_{(1,39)}=26.5, p<0.001\right)$ and error rates; $\left.F_{(1,39)}=27.2, p<0.001\right)$. Reaction times were longer for incongruent (mean \pm SD: $673 \pm 103 \mathrm{~ms})$ than for congruent movements $(626 \pm 128 \mathrm{~ms})\left(t_{(39)}=5.12, p<0.001\right.$ with an effect size of $d=0.4$, CI $[0.22,1.03]$, calculated as $\left.\left.(\mathrm{M} 1-\mathrm{M} 2) / \mathrm{sd}_{\text {pooled }}\right]\right)$. Participants also made more errors during incongruent (94.3 \pm $4.43 \%$ correct) than congruent blocks (97.1 $\pm 2.96 \%$ correct) $\left(t_{(39)}=5.21, p<0.001\right.$; effect size $d=0.7$, CI $\left.[0.27,1.2]\right)$. In addition to these interaction effects, there were main effects of movement $\left(F_{(1,39)}=32.9, p<0.001\right)$ and emotion $\left(F_{(1,39)}=7.7\right.$, $p=0.008$ ) on reaction times, but not on error rates (both $p>$ 0.15). Approach movements were faster $(626 \pm 106 \mathrm{~ms})$ than avoid movements $(661 \pm 111 \mathrm{~ms})$, and reactions to happy faces $(635 \pm 103 \mathrm{~ms})$ were faster than to angry faces $(652 \pm 114 \mathrm{~ms})$. Interaction effects are depicted in Figure $1 B$. These effects illus- 


\section{Resting state connectivity}

A
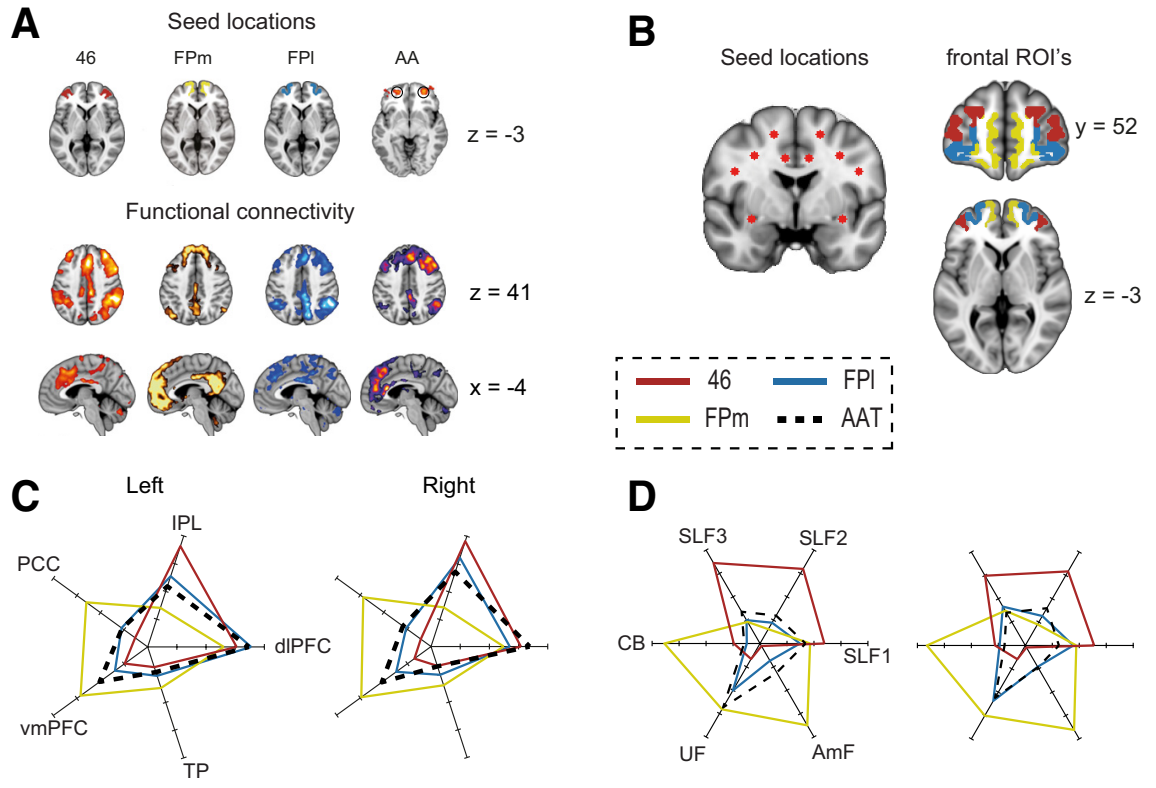

Figure 2. AA task effects are localized within the connectivity-defined FPI. $\boldsymbol{A}$, Seed regions and whole-brain resting-state connectivity for area 46, FPm, FPI, and for the cluster showing AA task effects. B, Seed locations for tractography in five large white matter tracts (AmF seed not shown) and masks for the regions FPI, FPm, and area 46. C, Resting-state connectivity fingerprints of the aPFC and AA regions, separated for left and right ROIs. D, Connectivity fingerprints for tractography of aPFC and the AA regions, separated for left and right ROIs. IPL, Intraparietal lobule; TP, temporal pole; CB, cingulum bundle; AmF, amygdalofugal.

trate the behavioral cost of applying cognitive control over prepotent habitual emotional action tendencies elicited by socialemotional stimuli.

In the replication sample there were similar interaction effects between emotion and movement for reaction times $\left(F_{(1,49)}=\right.$ $49.7, p<0.001)$ and error rates $\left(F_{(1,49)}=37.5, p<0.001\right)$. Reaction times were longer for incongruent $(676 \pm 133 \mathrm{~ms})$ than congruent $(627 \pm 107 \mathrm{~ms})\left(t_{(49)}=7, p<0.001\right.$; effect size $d=$ 0.4 , CI $[0.15,0.97])$. The replication sample also made more errors during incongruent $(94.5 \pm 3.8 \%$ correct $)$ than in congruent trials $(96.8 \pm 2.9 \%$ correct $)\left(t_{(49)}=6.13 p<0.001, d=0.68\right.$, [CI $0.11,1.25])$. In addition to these effects of interest, there were again main effects of movement $\left(F_{(1,49)}=26.6, p<0.001\right)$ and emotion $\left(F_{(1,49)}=7.5, p=0.008\right)$ on reaction times, but not on error rates (both $p>0.23$ ). Approach movements were faster $(641 \pm 122 \mathrm{~ms})$ than avoid movements $(661 \pm 127 \mathrm{~ms})$ and reactions to happy faces $(645 \pm 124 \mathrm{~ms})$ were faster than to angry faces $(657 \pm 125 \mathrm{~ms})$.

\section{AA task: controlling emotional actions elicits frontal and parietal activation}

Building on previous evidence (Volman et al., 2011a; Tyborowska et al., 2016), we expected that control over social emotional action tendencies increases aPFC activity. This study tests whether this activity can be localized to the FPl. We found clusters of increased activity over frontal areas during incongruent compared with congruent trials, whole-brain cluster-level corrected for multiple comparisons (cluster-forming threshold of $Z>2.3$ with corrected cluster threshold of $p<0.05$ ). Overlaying the frontal activity profiles on the mask created by Neubert et al. (2014) confirmed activity in FPl (Fig. 1C). In addition, we observed clusters of increased activity in bilateral intraparietal lobule, an area strongly connected to the aPFC (Mars et al., 2011), bilateral insula/inferior frontal gyrus $[34,26,-6]$, bilateral area $8 \mathrm{~A}$

\section{Relation between AA-related activity and functional} fingerprints of FPl (resting-state connectivity)

To assess whether the aPFC activity evoked during emotional action control falls within FPl (Neubert et al., 2014), we compared restingstate connectivity profiles of area $46, \mathrm{FPl}$, and FPm with the connectivity profile of the aPFC clusters showing a congruency effect (MNI: $[24,50,-4]$ and $[-24,52,-4])$. We tested whether the connectivity fingerprints of the three prefrontal areas differed from the connectivity profile of the AA-related clusters (Fig. 2).

Qualitative inspection of the resting-state fingerprints showed relatively similar connectivity profiles for area 46 and FPl. Both are strongly correlated with the intraparietal sulcus and dIPFC; however, FPl shows stronger connectivity to the PCC. Functional connectivity of FPm differed quite strongly from both area 46 and $\mathrm{FPl}$, showing strongest connectivity with ventromedial prefrontal areas (vmPFC), PCC, and temporal pole (Fig. $2 A, C$ ). These results corroborate earlier reports on resting-state connectivity fingerprints for these areas (Neubert et al., 2014; Mars et al., 2016; Hartogsveld et al., 2018).

Comparing connectivity profiles between the three areas in aPFC with the connectivity profile extracted from the AA-related region showed that its fingerprint differed significantly from that of area 46 (left hemisphere: $p<0.001$; right hemisphere: $p<0.001$ ), and from that of area FPm (right hemisphere: $p<0.001$; left hemisphere: $p<$ 0.001 ), but not from the connectivity fingerprint of area FPl (left hemisphere: $p=0.87$; right hemisphere: $p=0.075$ ). This result provides evidence that control of social emotional action tendencies involves the FPl.

\section{Relation between AA-related activity and structural fingerprints of FPI (DWI connectivity)}

In addition to resting-state connectivity, we created connectivity profiles based on white matter tractography. We placed seeds 
A

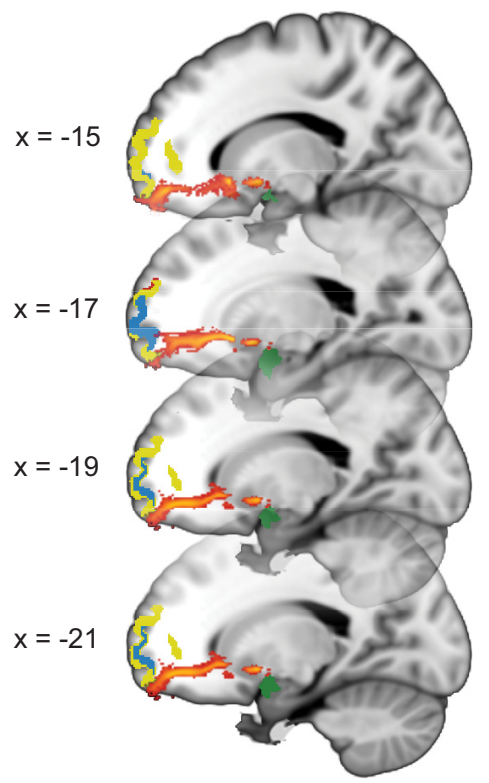

B
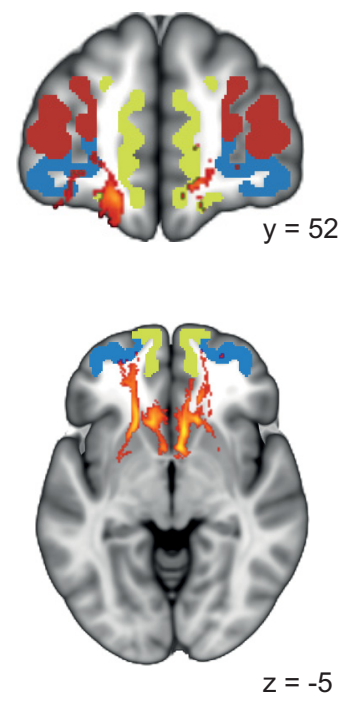

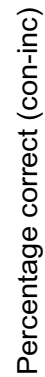

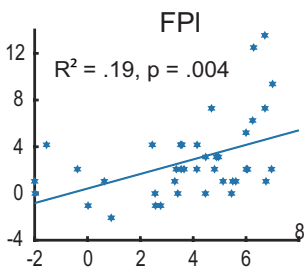

C: replication sample

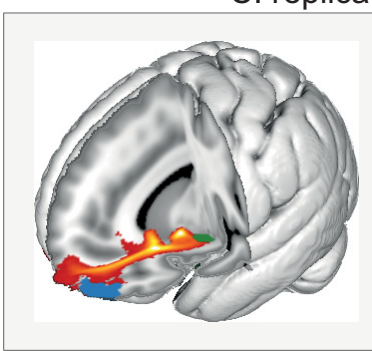

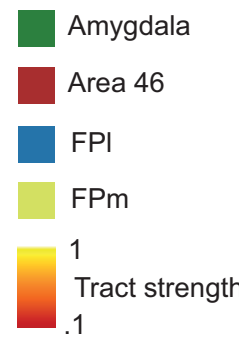

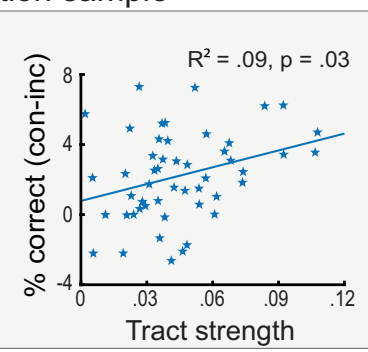

Figure 3. Amygdalofugal connections with FPI correlate with AA task performance. $A$, Illustration of the reconstructed tract that leads from the amygdala to the aPFC. This tract shows strongest connectivity with medial prefrontal areas, as can be expected given earlier studies. $\boldsymbol{B}$, The number of times the tract ends in FPI correlates with behavioral congruency in error rates on the AA task $(r=0.44, p=0.0044)$, suggesting that the amygdalofugal pathway is involved in mediating FPl-amygdala functional interactions during social-emotional control. $C$, The reconstructed tract and correlation between behavioral congruency and tract strength with FPI in the replication sample.

in five large white matter tracts (Fig. $2 B$ ), performed probabilistic tractography to the rest of the hemisphere, and counted how often each tract ended in each of the three regions in aPFC (Fig. $2 B$ ). We used a similar procedure for amygdalofugal connectivity (see Materials and Methods).

Comparing connectivity profiles of the aPFC regions to these five major white matter tracts and amygdalofugal connectivity showed relatively strong connectivity between area 46 and the three superior longitudinal fasciculi (SLF1, SLF2, and SLF3; Fig. $2 D$ ). FPm is most strongly connected to the cingulum bundle, UF, and amygdalofugal pathways. FPl is less strongly connected to the tracts leading outside the frontal cortex, and more strongly connected to the UF, which leads to the temporal pole (among other regions). The AA-related regions are also more strongly connected to the UF. These results are shown in Figure $2 D$ and match earlier reports on connectivity of aPFC regions (Neubert et al., 2014; Mars et al., 2016; Hartogsveld et al., 2018).

Comparing connectivity profiles between the three areas in aPFC with the connectivity profile extracted from the AA region showed that the fingerprint of the AA region differed significantly from that of area 46 (left hemisphere: $p<0.001$; right hemisphere: $p<0.001$ ), and from that of area FPm (right hemisphere: $p<0.001$; left hemisphere: $p<0.001$ ), but not from the connec- tivity fingerprint of area FPl (left hemisphere: $p=0.98$; right hemisphere: $p=1$ ). This result corroborates the resting-state results, providing additional evidence that the FPl is one of the regions involved in controlling social emotional action tendencies.

\section{FPl-amygdalofugal connectivity partly explains performance on the AA task}

To identify an anatomical pathway through which the FPl could support emotional behavior, we tested whether intersubject variability in the strength of FPl structural connectivity mediated by the amygdalofugal connections predicts successful performance on the AA task. On average, the FPm is more strongly connected to amygdalofugal pathways than the FPl and area 46 (Fig. 3A), in line with earlier studies showing both functionally and anatomically stronger connections between limbic areas and medial prefrontal regions (Ghashghaei and Barbas, 2002; Neubert et al., 2014; Folloni et al., 2019). However, individual variability in the connectivity of FPl and FPm confirms that the former region is more strongly involved in emotional action control than the latter. Namely, there was a significant correlation between tract strength and AA congruency effect on percentage correct (Spearman's $\left.r_{(38)}=0.44, p=0.004\right)$ for the FPl connections but not for $\operatorname{FPm}\left(r_{(38)}=0.24, p=0.13\right)$ and area $46\left(r_{(38)}=0.3, p=0.062\right)$ 
(Fig. 3B). However, these correlations did not differ from one another (both comparisons $p>0.3$ ). The direction of the FPlbehavioral correlation indicated that stronger connectivity was related to relatively worse performance when having to exert control. There were no significant correlations between tract strength and congruency effect on reaction time (all $p>0.09$ ). The congruency-FPl correlation for percentage correct survives Bonferroni correction for multiple comparisons over the eight correlations computed between behavioral congruency and amygdalofugal-FPl connectivity and the two control correlations described below.

To facilitate data interpretation, we conducted two additional exploratory control analyses. First, to exclude that the correlation between amygdalofugal-FPl connectivity and AA performance could be better explained by connections between amygdala and FPl through the UF (Petrides and Pandya, 2007) or trait anxiety (Kim and Whalen, 2009), we correlated UF-FPl connectivity with AA performance, and amygdalofugal-FPl connectivity with scores on the State Anxiety Inventory. None of these analyses yielded significant correlations (all $p>0.58$ ). Nor did the correlation between amygdalofugal-FPl connections and behavioral congruency change when controlling for state anxiety or UF-FPl connectivity.

Second, to explore whether FPl-amygdalofugal connectivity would also influence functional connectivity between these two areas, we performed a psychophysiological interaction analysis with bilateral amygdalae as seed region and the strength of the connection with $\mathrm{FPl}$ as covariate. This analysis showed a negative correlation between tract strength and amygdala-FPl functional connectivity during the task, when using a small volume correction over bilateral FPl ( $p=0.019$, FWE cluster corrected; cluster location MNI [ -32, 50, 6], cluster size: 20 voxels), meaning that, with increased tract strength, there is more negative connectivity between the FPl and amygdala, which might be interpreted as increased regulation of amygdala by the FPl (Volman et al., 2013).

Finally, given the novelty of the role of the amygdalofugal path in emotion control, we tested whether the correlation between behavioral congruency and amygdalofugal connectivity would replicate in an independent sample of 50 participants. The positive relationship between the congruency effect in percentage correct on the AA task and amygdalofugal-FPl connections was confirmed (Pearson's $r_{(48)}=0.3, p=0.03$; Spearman's $r_{(48)}=$ $0.28, p=0.05$; Fig. $3 C$ ), further substantiating this finding.

\section{Discussion}

The present study anatomically qualifies the involvement of the aPFC in control of action tendencies elicited by emotional faces. There are two main findings. First, structural and functional connectivity fingerprints of the FPl (Neubert et al., 2014) closely match the fingerprints of the aPFC territory recruited when emotional action control is required. Second, the strength of FPl structural connectivity with the amygdala accounts for a substantial portion of interindividual variation in emotional action regulation abilities. These findings provide evidence for a precise cortical region $(\mathrm{FPl})$ and a structural pathway (the ventral amygdalofugal bundle) through which a cognitively complex form of emotional regulation might be implemented in the human brain.

\section{FPl supports emotional action control}

Previous work has repeatedly shown that the aPFC is involved in controlling emotional behavior, and that it is capable of doing so by influencing downstream activity in the amygdala (Volman et al., 2013; Tyborowska et al., 2016), motor cortex, and parietal areas (Volman et al., 2011a; Bramson et al., 2018). Here, we extend this knowledge by showing that the aPFC contribution to emotional action control arises from a precise anatomical structure: the FPl (Neubert et al., 2014). This finding is based on the connectivity fingerprint of the FPl. This anatomical metric also provides clues to the information received by the $\mathrm{FPl}$, and the influence it can exert on other brain structures (Mars et al., 2018). The FPl, differently from neighboring areas FPm and 46, is connected to both medial and lateral circuits with parietal, cingulate, and temporal regions (Fig. 2). The peculiar pattern of FPl connectivity fits with the known supramodal regulatory role of this area during social-emotional control (Volman et al., 2011a, 2013; Koch et al., 2020), and with the notion that social-emotional regulation involves access to both egocentric value of emotionally laden actions, as well as social consequences of those actions (Koch et al., 2018).

This study was designed to add functional specificity to those anatomically grounded inferences on aPFC contributions to emotional action regulation. The observations of this study link emotional action regulation to several cognitive processes that rely on the $\mathrm{FPl}$, for example, concurrent monitoring of current and alternative goals (Burgess et al., 2007; Badre and D'Esposito, 2009; Mansouri et al., 2017), cognitive exploration (Daw et al., 2006; Zajkowski et al., 2017), counterfactual reasoning (Boorman et al., 2009; Koechlin, 2016), metacognition (Fleming et al., 2014; Shekhar and Rahnev, 2018), and relational reasoning (Vendetti and Bunge, 2014; Hartogsveld et al., 2018). More precisely, it becomes relevant to test whether the $\mathrm{FPl}$ contribution to emotional action control is linked to the online maintenance and evaluation of alternative goals or counterfactual regulation strategies (Sheppes et al., 2014; Koch et al., 2018); or whether the cognitive demands of emotional action control and their inputoutput connectivity segregate this faculty from other known FPl functions.

\section{Amygdala-FPl projections modulate control over emotional action}

This study shows that control over emotional actions is negatively associated with the strength of amygdala-FPl connections, with connections between amygdala and FPl explaining between 10\% and $20 \%$ of the variance on the AA task. This result was confirmed in an independent replication sample. On the assumption that the amygdala-FPl connectivity indexed in this study captures the amygdalofugal pathway (Folloni et al., 2019), that is, the main efferent pathways from amygdala to frontal cortex (Nolte, 1999; Ghashghaei and Barbas, 2002; Noback et al., 2005; Krüger et al., 2015; Kamali et al., 2016), this finding suggests that stronger amygdala projections to the FPl impair emotional regulation. Namely, during incongruent trials, stronger amygdala afferences to the FPl could lead to stronger automatic action tendencies or heightened emotional vigilance and thus more errors when those tendencies need to be rapidly overruled. This observation fits with previous imaging work showing that successful emotional control reduces bottom-up effective connectivity between amygdala and aPFC (Volman et al., 2013). Moreover, it suggests that top-down regulatory FPl efferences might reach the amygdala through other fiber bundles, for example, the uncinate fasciculus (Petrides and Pandya, 2007; Folloni et al., 2019); or through other regions, for example, the nucleus accumbens (Wager et al., 2008) or the orbitofrontal cortex (Ray and Zald, 2012). 
The relation between amygdala-FPl connectivity strength and emotional action control observed in this study is surprisingly large compared with the magnitude of previous structure-function correlations in the same anatomical regions (Jung et al., 2018). We suspect that the large effect size is a consequence of considering the anatomical differences between fiber bundles connecting the amygdala to the PFC, and in particular the uniquely human configuration of that connectivity, since anthropoid monkeys do not possess a functional homolog of the human FPl (Semendeferi et al., 2011; Neubert et al., 2014; Mars et al., 2016). Future tests of this potential functional-anatomical dissociation between bottom-up and top-down amygdala-FPl connectivity might also help to clarify the heterogeneity of previous findings concerning the relation between amygdala-prefrontal connectivity and state-anxiety measures (Kim and Whalen, 2009; Clewett et al., 2014).

\section{Interpretational issues}

It could be argued that the regulation required in the AA task is just an instance of inhibitory control with social stimuli, and that regulation in this task could be accomplished by suppressing automatic emotional action tendencies (Etkin et al., 2006; Aron et al., 2014). Several studies have shown that this is not a viable option. First, when participants respond to the gender rather than the emotion of the face, both behavioral (congruency effect) and neural correlates of emotional control (FPl activation) are extinguished (Roelofs et al., 2009a; Volman et al., 2011b). This illustrates that the regulation indexed by the AA task crucially depends on the interaction between emotional percepts and action selection, rather than on overriding conflict contained in the stimulus itself (e.g., emotional Stroop task, which is supported by different neural circuitry) (Etkin et al., 2011, 2015). Second, performance on the AA task is specifically altered in patients with disrupted social-emotional regulation (Heuer et al., 2007; van Peer et al., 2009; von Borries et al., 2012). For instance, aggression-related psychopathologies, such as psychopathy, are marked by reduction of avoidance responses, specific to angry faces, an effect related to measures of social aggression (von Borries et al., 2012). In contrast, patients with social anxiety disorder show a consistent increase of avoidance responses, specific to angry faces (Roelofs and Cremers, 2005; Roelofs et al., 2009b; Heuer et al., 2007). In addition, socially relevant hormones, such as testosterone (Tyborowska et al., 2016), cortisol (Volman et al., 2011b), and oxytocin (Radke et al., 2017), influence performance of the AA task in healthy participants. Most critically, the aPFC congruency effect measured during the social AA task is predictive of subjective, physiological, and hormonal responses to a real-life social stressor (Kaldewaij et al., 2019b). In sum, these studies highlight the validity of the AA task for indexing regulation of emotional actions in social contexts.

In principle, the relation between strength of amygdala-FPl connectivity and behavioral emotional control could be explained by a third factor (e.g., trait anxiety). However, the tract strength between amygdala and FPl did not correlate with trait anxiety, and the correlation between behavioral congruency and tract strength persisted when controlling for anxiety. This observation confirms that, in healthy participants, aPFC activity during the AA task is orthogonal to trait anxiety effects (Bramson et al., 2018), in contrast to known trait anxiety effects on amygdalavmPFC connectivity (Kim and Whalen, 2009).

It is not easy to acquire reliable fMRI signals from anterior prefrontal regions (Hutton et al., 2002). Therefore, we used magnetic field corrections to restore signal distortions around the frontal pole (Jezzard and Balaban, 1995). The resting-state connectivity profiles closely matched those of earlier studies that used different MRI protocols (Neubert et al., 2014; Mars et al., 2016), arguing against the possibility that the results reported here can be attributed to signal distortions. The first sample of participants consisted of males only, and the replication sample was predominantly male. Whereas behavioral and neural effects in the AA task have been shown in mixed and female only samples as well (Radke et al., 2015; Tyborowska et al., 2016; Bertsch et al., 2018), we recruited only males to avoid having to control for differences in social hormones, such as testosterone and cortisol, which would require a larger sample of participants. These hormones are known to influence behavioral and neural responses on the AA task (van Peer et al., 2007; Volman et al., 2011b; Kaldewaij et al., 2019a). Future studies could explore similarities and potential differences in emotional processing between sexes (Domes et al., 2010; Whittle et al., 2011).

In conclusion, this study provides anatomical evidence supporting the involvement of the FPl, partly via the ventral amygdalofugal pathway, in the regulation of emotional behavior (Koch et al., 2018). The findings have implications for structuring mechanism-based interventions in psychopathologies characterized by altered emotional control abilities, for example, anxiety disorders and psychopathy (Volman et al., 2016). The findings are also relevant for understanding the neurobiological and cognitive complexities underlying rule-based regulation of action tendencies, arguably a crucial prerequisite for the development of human cumulative culture (Hare, 2017; Whiten, 2017).

\section{References}

Andersson JL, Sotiropoulos SN (2016) An integrated approach to correction for off-resonance effects and subject movement in diffusion MR imaging. Neuroimage 125:1063-1078.

Andersson JL, Skare S, Ashburner J (2003) How to correct susceptibility distortions in spin-echo echo-planar images: application to diffusion tensor imaging. Neuroimage 20:870-888.

Aron AR, Robbins TW, Poldrack RA (2014) Inhibition and the right inferior frontal cortex: one decade on. Trends Cogn Sci 18:177-185.

Badre D, D'Esposito M (2009) Is the rostro-caudal axis of the frontal lobe hierarchical? Nat Rev Neurosci 10:659-669.

Beckmann CF, Smith SM (2004) Probabilistic independent component analysis for functional magnetic resonance imaging. IEEE Trans Med Imaging 23:137-152.

Behrens TE, Berg HJ, Jbabdi S, Rushworth MF, Woolrich MW (2007) Probabilistic diffusion tractography with multiple fibre orientations: what can we gain? Neuroimage 34:144-155.

Bertsch K, Volman I, Roelofs K, Herpertz SC, Müller LE (2017) Social emotional leaning and behavioral tendencies in high and low socially anxious men and women. Psychoneuroendocrinology 83:49-57.

Bertsch K, Roelofs K, Roch PJ, Ma B, Hensel S, Herpertz SC, Volman I (2018) Neural correlates of emotional action control in anger-prone women with borderline personality disorder. J Psychiatry Neurosci 43:170102.

Boorman ED, Behrens TE, Woolrich MW, Rushworth MF (2009) How green is the grass on the other side? Frontopolar cortex and the evidence in favor of alternative courses of action. Neuron 62:733-743.

Bramson B, Jensen O, Toni I, Roelofs K (2018) Cortical oscillatory mechanisms supporting the control of human social-emotional actions. J Neurosci 38:5739-5749.

Buhle JT, Silvers JA, Wager TD, Lopez R, Onyemekwu C, Kober H, Weber J, Ochsner KN (2014) Cognitive reappraisal of emotion: a meta-analysis of human neuroimaging studies. Cereb Cortex 24:2981-2990.

Burgess PW, Dumontheil I, Gilbert SJ (2007) The gateway hypothesis of rostral prefrontal cortex (area 10) function. Trends Cogn Sci 11:290-298.

Clewett D, Bachman S, Mather M (2014) Age-related reduced prefrontalamygdala structural connectivity is associated with lower trait anxiety. Neuropsychology 28:631-642.

Daw ND, O’Doherty JP, Dayan P, Seymour B, Dolan RJ (2006) Cortical substrates for exploratory decisions in humans. Nature 441:876-879. 
Desikan RS, Ségonne F, Fischl B, Quinn BT, Dickerson BC, Blacker D, Buckner RL, Dale AM, Maguire RP, Hyman BT (2006) An automated labeling system for subdividing the human cerebral cortex on MRI scans into gyral based regions of interest. Neuroimage 31:968-980.

Domes G, Schulze L, Böttger M, Grossmann A, Hauenstein K, Wirtz PH, Heinrichs M, Herpertz SC (2010) The neural correlates of sex differences in emotional reactivity and emotion regulation. Hum Brain Mapp $31: 758-769$.

Eklund A, Nichols TE, Knutsson H (2015) Can parametric statistical methods be trusted for fMRI based group studies? Proc Natl Acad Sci U S A 113:7900-7905.

Etkin A, Egner T, Peraza DM, Kandel ER, Hirsch J (2006) Resolving emotional conflict: a role for the rostral anterior cingulate cortex in modulating activity in the amygdala. Neuron 51:871-882.

Etkin A, Egner T, Kalisch R (2011) Emotional processing in anterior cingulate and medial prefrontal cortex. Trends Cogn Sci 15:85-93.

Etkin A, Büchel C, Gross JJ (2015) The neural bases of emotion regulation. Nat Rev Neurosci 16:693-700.

Fleming SM, Ryu J, Golfinos JG, Blackmon KE (2014) Domain-specific impairment in metacognitive accuracy following anterior prefrontal lesions. Brain 137:2811-2822.

Folloni D, Sallet J, Khrapitchev AA, Sibson N, Verhagen L, Mars RB (2019) Dichotomous organization of amygdala/temporal-prefrontal bundles in both humans and monkeys. Elife 8:e47175.

Frijda NH, Ridderinkhof KR, Rietveld E (2014) Impulsive action: emotional impulses and their control. Front Psychol 5:518.

Ghashghaei HT, Barbas H (2002) Pathways for emotion: interactions of prefrontal and anterior temporal pathways in the amygdala of the rhesus monkey. Neuroscience 115:1261-1279.

Ghashghaei HT, Hilgetag CC, Barbas H (2007) Sequence of information processing for emotions based on the anatomic dialogue between prefrontal cortex and amygdala. Neuroimage 34:905-923.

Griffanti L, Douaud G, Bijsterbosch J, Evangelisti S, Alfaro-Almagro F, Glasser MF, Duff EP, Fitzgibbon S, Westphal R, Carone D, Beckmann CF, Smith SM (2017) Hand classification of fMRI ICA noise components. Neuroimage 154:188-205.

Hare B (2017) Survival of the friendliest: homo sapiens evolved via selection for prosociality. Annu Rev Psychol 68:155-186.

Hartogsveld B, Bramson B, Vijayakumar S, van Campen AD, Marques JP, Roelofs K, Toni I, Bekkering H, Mars RB (2018) Lateral frontal pole and relational processing: activation patterns and connectivity profile. Behav Brain Res 355:2-11.

Heuer K, Rinck M, Becker ES (2007) Avoidance of emotional facial expressions in social anxiety: the approach-avoidance task. Behav Res Ther 45:2990-3001.

Hutton C, Bork A, Josephs O, Deichmann R, Ashburner J, Turner R (2002) Image distortion correction in fMRI: a quantitative evaluation. Neuroimage 16:217-240.

Jenkinson M, Bannister P, Brady M, Smith S (2002) Improved optimization for the robust and accurate linear registration and motion correction of brain images. Neuroimage 17:825-841.

Jezzard P, Balaban RS (1995) Correction for geometric distortion in echo planar images from B0 field variations. Magn Reson Med 34:65-73.

Jung WH, Lee S, Lerman C, Kable JW (2018) Amygdala functional and structural connectivity predicts individual risk tolerance. Neuron 98:394-404.

Kaldewaij R, Koch SB, Zhang W, Hashemi MM, Klumpers F, Roelofs K (2019a) High endogenous testosterone levels are associated with diminished neural emotional control in aggressive police recruits. Psychol Sci 8:1161-1173.

Kaldewaij R, Koch SB, Zhang W, Hashemi MM, Klumpers F, Roelofs K (2019b) Frontal control over automatic emotional action tendencies predicts acute stress responsivity. Biol Psychiatry Cogn Neurosci Neuroimaging 4:975-983.

Kamali A, Sair HI, Blitz AM, Riascos RF, Mirbagheri S, Keser Z, Hasan KM (2016) Revealing the ventral amygdalofugal pathway of the human limbic system using high spatial resolution diffusion tensor tractography. Brain Struct Funct 221:3561-3569.

Kim MJ, Whalen PJ (2009) The structural integrity of an amygdalaprefrontal pathway predicts trait anxiety. J Neurosci 29:11614-11618.

Koch SB, Mars RB, Toni I, Roelofs K (2018) Emotional control, reappraised. Neurosci Biobehav Rev 95:528-534.

Koch SBJ, Galli A, Volman I, Kaldewaij R, Toni I, Roelofs K (2020) Neural control of emotional actions in response to affective vocalizations. J Cogn Neurosci:1-12.

Koechlin E (2016) Prefrontal executive function and adaptive behavior in complex environments. Curr Opin Neurobiol 37:1-6.

Krüger O, Shiozawa T, Kreifelts B, Scheffler K, Ethofer T (2015) Three distinct fiber pathways of the bed nucleus of the stria terminalis to the amygdala and prefrontal cortex. Cortex 66:60-68.

Langner R, Leiberg S, Hoffstaedter F, Eickhoff SB (2018) Towards a human self-regulation system: common and distinct neural signatures of emotional and behavioural control. Neurosci Biobehav Rev 90:400-410.

Mansouri FA, Koechlin E, Rosa MG, Buckley MJ (2017) Managing competing goals: a key role for the frontopolar cortex. Nat Rev Neurosci 18:645-657.

Mars RB, Jbabdi S, Sallet J, O'Reilly JX, Croxson PL, Olivier E, Noonan MP, Bergmann C, Mitchell AS, Baxter MG, Behrens TE, Johansen-Berg H, Tomassini V, Miller KL, Rushworth MF (2011) Diffusion-weighted imaging tractography-based parcellation of the human parietal cortex and comparison with human and macaque resting-state functional connectivity. J Neurosci 31:4087-4100.

Mars RB, Verhagen L, Gladwin TE, Neubert FX, Sallet J, Rushworth MF (2016) Comparing brains by matching connectivity profiles. Neurosci Biobehav Rev 60:90-97.

Mars RB, Passingham RE, Jbabdi S (2018) Connectivity fingerprints: from areal descriptions to abstract spaces. Trends Cogn Sci 22:1026-1037.

Morawetz C, Bode S, Derntl B, Heekeren HR (2017) The effect of strategies, goals and stimulus material on the neural mechanisms of emotion regulation: a meta-analysis of fMRI studies. Neurosci Biobehav Rev 72: $111-128$.

Neubert FX, Mars RB, Thomas AG, Sallet J, Rushworth MF (2014) Comparison of human ventral frontal cortex areas for cognitive control and language with areas in monkey frontal cortex. Neuron 81:700-713.

Noback CR, Ruggiero DA, Demarest RJ, Strominger NL (2005) The human nervous system: structure and function. New York: Springer.

Nolte J (1999) The human brain: an introduction to its functional anatomy. St. Louis: Mosby.

Passingham RE, Stephan KE, Kötter R (2002) The anatomical basis of functional localization in the cortex. Nat Rev Neurosci 3:606-616.

Petrides M, Pandya DN (2007) Efferent association pathways from the rostral prefrontal cortex in the macaque monkey. J Neurosci 27:1157311586.

Radke S, Roelofs K, de Bruijn ER (2013) Acting on anger: social anxiety modulates approach-avoidance tendencies after oxytocin administration. Psychol Sci 24:1573-1578.

Radke S, Volman I, Mehta P, van Son V, Enter D, Sanfey A, Toni I, de Bruijn ER, Roelofs K (2015) Testosterone biases the amygdala toward social threat approach. Sci Adv 1:e1400074.

Radke S, Volman I, Kokal I, Roelofs K, de Bruijn ER, Toni I (2017) Oxytocin reduces amygdala responses during threat approach. Psychoneuroendocrinology 79:160-166.

Ray RD, Zald DH (2012) Anatomical insights into the interaction of emotion and cognition in the prefrontal cortex. Neurosci Biobehav Rev 36: 479-501.

Ridderinkhof KR (2017) Emotion in action: a predictive processing perspective and theoretical synthesis. Emot Rev 9:319-325.

Roelofs K, Cremers HR (2015) Cortical-amygdala decoupling during stress in patients with social anxiety disorder. Biol Psychiatry 77:S298.

Roelofs K, Minelli A, Mars RB, van Peer J, Toni I (2009a) On the neural control of social emotional behavior. Soc Cogn Affect Neurosci 4:50-58.

Roelofs K, van Peer J, Berretty E, de Jong P, Spinhoven P, Elzinga BM (2009b) Hypothalamus-pituitary-adrenal axis hyperresponsiveness is associated with increased social avoidance behavior in social phobia. Biol Psychiatry 65:336-343.

Sallet J, Mars RB, Noonan MP, Neubert FX, Jbabdi S, O'Reilly JX, Filippini N, Thomas AG, Rushworth MF (2013) The organization of dorsal frontal cortex in humans and macaques. J Neurosci 33:12255-12274.

Semendeferi K, Teffer K, Buxhoeveden DP, Park MS, Bludau S, Amunts K, Travis K, Buckwalter J (2011) Spatial organization of neurons in the frontal pole sets humans apart from great apes. Cereb Cortex 21:14851497.

Shekhar M, Rahnev D (2018) Distinguishing the roles of dorsolateral and anterior PFC in visual metacognition. J Neurosci 38:5078-5087.

Sheppes G, Scheibe S, Suri G, Radu P, Blechert J, Gross JJ (2014) Emotion 
regulation choice: a conceptual framework and supporting evidence. J Exp Psychol Gen 143:163-181.

Tillman RM, Stockbridge MD, Nacewicz BM, Torrisi S, Fox AS, Smith JF, Shackman AJ (2018) Intrinsic functional connectivity of the central extended amygdala. Hum Brain Mapp 39:1291-1312.

Tyborowska A, Volman I, Smeekens S, Toni I, Roelofs K (2016) Testosterone during puberty shifts emotional control from pulvinar to anterior prefrontal cortex. J Neurosci 36:6156-6164.

Tzourio-Mazoyer N, Landeau B, Papathanassiou D, Crivello F, Etard O, Delcroix N, Mazoyer B, Joliot M (2002) Automated anatomical labeling of activations in SPM using a macroscopic anatomical parcellation of the MNI MRI single-subject brain. Neuroimage 15:273-289.

van Peer JM, Roelofs K, Rotteveel M, van Dijk JG, Spinhoven P, Ridderinkhof KR (2007) The effects of cortisol administration on approach-avoidance behavior: an event-related potential study. Biol Psychol 76:135-146.

van Peer JM, Spinhoven P, van Dijk JG, Roelofs K (2009) Cortisol-induced enhancement of emotional face processing in social phobia depends on symptom severity and motivational context. Biol Psychol 81:123-130.

Vendetti MS, Bunge SA (2014) Evolutionary and developmental changes in the lateral frontoparietal network: a little goes a long way for higher-level cognition. Neuron 84:906-917.

Volman I, Roelofs K, Koch S, Verhagen L, Toni I (2011a) Anterior prefrontal cortex inhibition impairs control over social emotional actions. Curr Biol 21:1766-1770.

Volman I, Toni I, Verhagen L, Roelofs K (2011b) Endogenous testosterone modulates prefrontal-amygdala connectivity during social emotional behavior. Cereb Cortex 21:2282-2290.
Volman I, Verhagen L, den Ouden HE, Fernández G, Rijpkema M, Franke B, Toni I, Roelofs K (2013) Reduced serotonin transporter availability decreases prefrontal control of the amygdala. J Neurosci 33:8974-8979.

Volman I, von Borries AK, Bulten BH, Verkes RJ, Toni I, Roelofs K (2016) Testosterone modulates altered prefrontal control of emotional actions in psychopathic offenders. ENeuro 3:ENEURO-0107.

von Borries AK, Volman I, de Bruijn ER, Bulten BH, Verkes RJ, Roelofs K (2012) Psychopaths lack the automatic avoidance of social threat: relation to instrumental aggression. Psychiatry Res 200:761-766.

Wager TD, Davidson ML, Hughes BL, Lindquist MA, Ochsner KN (2008) Prefrontal-subcortical pathways mediating successful emotion regulation. Neuron 59:1037-1050.

Whiten A (2017) Culture extends the scope of evolutionary biology in the great apes. Proc Natl Acad Sci U S A 114:7790-7797.

Whittle S, Yücel M, Yap MB, Allen NB (2011) Sex differences in the neural correlates of emotion: evidence from neuroimaging. Biol Psychol 87: 319-333.

Woolrich MW, Ripley BD, Brady M, Smith SM (2001) Temporal autocorrelation in univariate linear modeling of fMRI data. Neuroimage 14:1370-1386.

Woolrich MW, Behrens TE, Beckmann CF, Jenkinson M, Smith SM (2004) Multilevel linear modelling for FMRI group analysis using Bayesian inference. Neuroimage 21:1732-1747.

Zajkowski WK, Kossut M, Wilson RC (2017) A causal role for right frontopolar cortex in directed, but not random, exploration. Elife 6:e27430. 